\title{
Combination of biomarkers for the detection of hepatocellular carcinoma
}

\author{
Malin Sternby Eilard ${ }^{1}$, Fredrik Åberg ${ }^{2}$ \\ ${ }^{1}$ Transplant Institute, Sahlgrenska University Hospital, Gothenburg, Sweden; ${ }^{2}$ Transplantation and Liver Surgery Clinic, Helsinki University \\ Hospital, Helsinki University, Helsinki, Finland \\ Correspondence to: Fredrik Åberg, MD, PhD. HUCH Meilahti Hospital, PB 372, 00029 HUS, Finland. Email: Fredrik.Aberg@helsinki.fi. \\ Comment on: Zhao S, Long M, Zhang X, et al. The diagnostic value of the combination of Golgi protein 73 , glypican-3 and alpha-fetoprotein in \\ hepatocellular carcinoma: a diagnostic meta-analysis. Ann Transl Med 2020;8:536.
}

Submitted Aug 09, 2020. Accepted for publication Aug 27, 2020.

doi: 10.21037/atm-20-5798

View this article at: http://dx.doi.org/10.21037/atm-20-5798

Globally, liver cancer was in 2018 the second most common cause of cancer-related death under the age of 80 (1), and almost $75 \%$ of cases were from Asia (1). The age-specific incidence of hepatocellular carcinoma (HCC) has been decreasing during recent years; nonetheless, due to growing and aging populations, the actual number of new cases annually is increasing (2). Hepatitis B and C along with alcohol remain the most common etiologic factors, while the incidence rate of HCC associated with non-alcoholic fatty liver disease (NAFLD) is rising rapidly (3). Diagnosis at early stages is crucial for successful curative treatment, but frequently a challenge due to the scarcity of symptoms until progression to late-stage HCC. Current screening strategies comprising ultrasound for high-risk patients (mainly those with cirrhosis) consume substantial amounts of resources, and sensitivity is often suboptimal (4). Despite diagnostic algorithms and predefined criteria such as the European Association for the Study of the Liver-American Association for the Study of Liver Diseases (EASL-AASLD) and the Liver Imaging Reporting and Data System (LIRADS) (5), multiple parenchymal changes in cirrhosis makes the interpretation of radiology difficult.

Serum biomarkers emerge as attractive diagnostic tools as they are based on simple blood samples and interpretation is straightforward given that cut-offs with both high sensitivity and specificity can be defined. For decades, alpha fetoprotein (AFP) has been the most common serum biomarker for HCC. Initially, AFP was used as a diagnostic tool, but the high proportion of early-stage HCC with normal AFP levels, in combination with improved radiology, led to the removal of AFP from modern diagnostic flow charts (6). More recently, AFP has regained a role in posttreatment surveillance and liver transplantation selection, based on the correlation of AFP with vascular invasion and tumor differentiation grade $(6,7)$. Improved sensitivity has also been reported for AFP when added to ultrasound surveillance (4). Despite its wide use in clinical practice, no consensus has yet been reached regarding the optimal AFP cut-off levels in different settings (8). Novel biomarkers such as Des-gamma-carboxy prothrombin (DCP) and AFP-L3 (the isoform of AFP bound to lectin Lens culinaris agglutinin) have been investigated as alternatives or complements to $\operatorname{AFP}(6,8)$, and combinations of these seem to improve diagnostic performance over AFP alone (9). Nonetheless, the literature is conflicting and no other biomarker than AFP has yet reached wide clinical practice. Genomic, epigenomic, transcriptomic and proteomic techniques have the potential to provide valuable input in the ongoing search for better biomarkers (10).

In this issue of Annals of Translational Medicine, Zhao and colleagues (11) report a meta-analysis of the value of combining Golgi protein 73 (GP73), glypican-3 (GPC-3) and AFP for the diagnosis of HCC. Based on 12 studies involving 919 HCC patients and 1,549 controls, the pooled sensitivity and specificity of the combination of all 3 serum biomarkers for detection of HCC were 0.91 and 0.84 , compared to 0.65 and 0.88 for AFP alone. Similarly, as a measure of diagnostic accuracy, the summary area-underthe-curve values were 0.95 for the biomarker combination and 0.88 for AFP alone.

GPC-3 is a heparan sulfate proteoglycan, with influence in HCC signaling such as the WNT and Hedgehog 
pathways (12). GPC-3 is widely expressed in the embryo, but not in healthy liver tissue or in non-malignant liver disease, whereas GPC-3 tissue expression has been reported in up to $70 \%$ of HCC cases (12). For tissue diagnostics, such as surgical specimens and biopsies, GPC-3 is an important tool included in clinical practice in combination with other markers (13). Increased expression of GPC-3 in serum from HCC patients has also been described (14), but results are conflicting $(12,15)$.

GP73 is a transmembrane protein with low expression in normal hepatocytes, but described to increase in both acute and chronic liver diseases (16). Improved sensitivity and specificity for HCC diagnosis with the use of serum GP73 compared to AFP has previously been reported (17-20), but again, findings have been conflicting $(16,21)$.

Zhao et al. performed an extensive literature search using the Web of Science, Cochrane Library, Embase, Chinese Biomedical Literature Database and China National Knowledge Infrastructure databases. The absence of PubMed from this list is, however, somewhat peculiar. Unfortunately, many of the studies included in the metaanalysis are not easily accessible to the international reader, which highlights the need to replicate these findings outside Asia.

While the process of literature review, quality assessment of the studies and meta-statistics are well-described, a number of clinical aspects remain obscure. First, the cutoff levels used for the various biomarkers in each study is not reported. Second, although the authors state that they included only patient cohorts with similar characteristics in order to avoid confounding, unfortunately, relevant characteristics of HCC cases and controls are not mentioned. For instance, it remains unclear how control subjects were selected and whether or not they had chronic liver disease or cirrhosis. Previous biomarker studies emphasize that it is often easy to distinguish HCC-patients from healthy individuals, whereas the distinction between HCC and non-malignant liver disease is more difficult, because biomarker levels may be increased in chronic liver disease even without HCC (19). Third, the need for serum biomarkers is highest in the early HCC stages; therefore, a description of tumor burden would have been crucial for a better understanding of diagnostic accuracy of the biomarker combination approach in a clinically relevant setting. Fourth, the exact algorithm of the biomarker combination, and whether the same algorithm was used in each study, remains unclear. If positivity of any one of the 3 biomarkers count for a diagnosis of HCC, then sensitivity will be high, while specificity will deteriorate. To increase specificity, one would usually demand positivity of 2 or 3 biomarkers, but then sensitivity will usually deteriorate. Fifth, also the setting to which the biomarker testing is intended for is important. For initial screening purposes, finding as many cases as possible is the priority, implying a need for high sensitivity of the test. In contrast, when the test is intended for final diagnosis, specificity would be the priority. Finally, considering that ultrasound with or without AFP is the screening strategy currently recommended by most societies, further studies are needed to compare the biomarker combination-approach to ultrasound +/- AFP, and to analyze the value of biomarkers when added to ultrasound.

Based on the findings by Zhao and colleagues, the approach of combining several serum biomarkers in the detection of HCC seems promising, but there is still a long way to go before this approach is ready for the clinic.

\section{Acknowledgments}

Funding: None.

\section{Footnote}

Provenance and Peer Review: This article was commissioned by the editorial office, Annals of Translational Medicine. The article did not undergo external peer review.

Conflicts of Interest: Both authors have completed the ICMJE uniform disclosure form (available at http://dx.doi. org/10.21037/atm-20-5798). Both authors have no conflicts of interest to declare.

Ethical Statement: The authors are accountable for all aspects of the work in ensuring that questions related to the accuracy or integrity of any part of the work are appropriately investigated and resolved.

Open Access Statement: This is an Open Access article distributed in accordance with the Creative Commons Attribution-NonCommercial-NoDerivs 4.0 International License (CC BY-NC-ND 4.0), which permits the noncommercial replication and distribution of the article with the strict proviso that no changes or edits are made and the original work is properly cited (including links to both the formal publication through the relevant DOI and the license). See: https://creativecommons.org/licenses/by-nc-nd/4.0/. 


\section{References}

1. International Agency for Research of Cancer. Lam F, Ervik M, Ferlay J, et al. Estimated number of incident cases and deaths worldwide, both sexes, ages 0-79. International Association of Cancer Registries; 2020. Available online: https://gco.iarc.fr/today/onlineanalysis-multi-bars? $\mathrm{v}=2018 \&$ mode $=$ cancer $\&$ mode population $=$ countries $\&$ population $=900 \&$ population $\mathrm{s}=900 \& \mathrm{key}=$ total $\&$ sex $=0$ \& cancer $=39 \&$ type $=0 \&$ stat istic $=5 \&$ prevalence $=0 \&$ population_group $=0 \&$ ages group $\% 5 \mathrm{~B} \% 5 \mathrm{D}=0 \&$ ages_group $\% 5 \mathrm{~B} \% 5 \mathrm{D}=15 \& \mathrm{nb}$ items=10\&group_cancer=1\&include_nmsc $=1 \&$ include_ nmsc_other=1\&type_multiple=\%257B\%2522inc\% $2522 \%$ 253 Afalse $\% 252$ C\% 2522mort \% 2522\%253Atrue \% 252C\% 2522 prev\%2522\%253Afalse $\% 257$ D\&orientation=horizon tal\&type_sort=0\&type_nb_items=\%257B\%2522top $\% 252$ $2 \% 253$ Atrue $\% 252$ C\% 2522bottom $\% 2522 \% 253$ Afalse $\% 25$ 7D\&population_group_globocan_id=

2. Global Burden of Disease Liver Cancer Collaboration, Akinyemiju T, Abera S, et al. The Burden of Primary Liver Cancer and Underlying Etiologies From 1990 to 2015 at the Global, Regional, and National Level: Results From the Global Burden of Disease Study 2015. JAMA Oncol 2017;3:1683-91.

3. Marengo A, Rosso C, Bugianesi E. Liver Cancer: Connections with Obesity, Fatty Liver, and Cirrhosis. Annu Rev Med 2016;67:103-17.

4. Tzartzeva K, Obi J, Rich NE, et al. Surveillance Imaging and Alpha Fetoprotein for Early Detection of Hepatocellular Carcinoma in Patients with Cirrhosis: A Meta-analysis. Gastroenterology 2018;154:1706-18.

5. Mitchell DG, Bruix J, Sherman M, et al. LI-RADS (Liver Imaging Reporting and Data System): Summary, Discussion, and Consensus of the LI-RADS Management Working Group and Future Directions. Hepatology 2015;61:1056-65.

6. Lai Q, Melandro F, Pinheiro RS, et al. Alpha-fetoprotein and novel tumor biomarkers as predictors of hepatocellular carcinoma recurrence after surgery: a brilliant star raises again. Int J Hepatol 2012;2012:893103.

7. Liu C, Xiao GQ, Yan LN, et al. Value of $\alpha$-fetoprotein in association with clinicopathological features of hepatocellular carcinoma. World J Gastroenterol 2013;19:1811-9.

8. Piñero F, Dirchwolf M, Pessôa MG. Biomarkers in Hepatocellular Carcinoma: Diagnosis, Prognosis and Treatment Response Assessment. Cells 2020;9:1370.

9. Yang JD, Addissie BD, Mara KC, et al. GALAD Score for Hepatocellular Carcinoma Detection in Comparison with Liver Ultrasound and Proposal of GALADUS Score. Cancer Epidemiol Biomarkers Prev 2019;28:531-8.

10. Liu XN, Cui DN, Li YF, et al. Multiple "Omics" databased biomarker screening for hepatocellular carcinoma diagnosis. World J Gastroenterol 2019;25:4199-212.

11. Zhao S, Long $M$, Zhang $X$, et al. The diagnostic value of the combination of Golgi protein 73, glypican-3 and alpha-fetoprotein in hepatocellular carcinoma: a diagnostic meta-analysis. Ann Transl Med 2020;8:536.

12. Guo M, Zhang H, Zheng J, et al. Glypican-3: A New Target for Diagnosis and Treatment of Hepatocellular Carcinoma. J Cancer 2020;11:2008-21.

13. Yao S, Zhang J, Chen H, et al. Diagnostic Value of Immunohistochemical Staining of GP73, GPC3, DCP, CD34, CD31, and Reticulin Staining in Hepatocellular Carcinoma. J Histochem Cytochem 2013;61:639-48.

14. Wu M, Liu Z, Li X, et al. Dynamic Changes in Serum Markers and Their Utility in the Early Diagnosis of All Stages of Hepatitis B-Associated Hepatocellular Carcinoma. Onco Targets Ther 2020;13:827-40.

15. Xu D, Su C, Sun L, et al. Performance of Serum Glypican 3 in Diagnosis of Hepatocellular Carcinoma: A metaanalysis. Ann Hepatol 2019;18:58-67.

16. Xia Y, Zhang Y, Shen M, et al. Golgi protein 73 and its diagnostic value in liver diseases. Cell Prolif 2019;52:e12538.

17. Marrero JA, Romano PR, Nikolaeva O, et al. GP73, a resident Golgi glycoprotein, is a novel serum marker for hepatocellular carcinoma. J Hepatol 2005;43:1007-12.

18. Fouad MA, Haleim EF, Azeim WF, et al. Golgi protein 73 versus alpha fetoprotein as a marker for hepatocellular carcinoma. Menoufia Med J 2016;29:152-9.

19. Ali OM, El Amin HA, Sharkawy YL, et al. Golgi Protein 73 versus Alpha-Fetoprotein as a New Biomarker in Early Diagnosis of Hepatocellular Carcinoma. Int J Gen Med 2020;13:193-200.

20. Dai M, Chen X, Liu X, et al. Diagnostic Value of the Combination of Golgi Protein 73 and Alpha-Fetoprotein in Hepatocellular Carcinoma: A Meta-Analysis. PLoS One 2015;10:e0140067.

21. Liu T, Yao M, Liu S, et al. Serum Golgi protein 73 is not a suitable diagnostic marker for hepatocellular carcinoma. Oncotarget 2017;8:16498-506.

Cite this article as: Sternby Eilard M, Åberg F. Combination of biomarkers for the detection of hepatocellular carcinoma. Ann Transl Med 2020;8(20):1283. doi: 10.21037/atm-20-5798 